\title{
Experimental Investigation of Fouling Remediation Strategies for Cross-flow Microfiltration of Fast Pyrolysis Bio-oil
}

\section{Supporting Information}

\section{Dillon Mazerolle ${ }^{1 *}$, Benjamin Bronson ${ }^{1}$, Boguslaw Kruczek²}

${ }^{1}$ Natural Resources Canada, CanmetENERGY, 1 Haanel Drive, Nepean, ON, K1A 1M1, Canada

${ }^{2}$ Department of Chemical and Biological Engineering, University of Ottawa, 161 Louis Pasteur Drive, Ottawa, ON, K1N 6N5, Canada

*dillon.mazerolle@canada.ca

\section{Design of Experimental Cross-flow Microfiltration System}

To study the microfiltration profiles of fast pyrolysis bio-oils for suspended char particulate (solids) and ash removal, a bench scale cross-flow microfiltration system was designed and assembled at CanmetENERGY-Ottawa. An image of the MRK III version of the experimental system which was used in this study, is below in Figure S1.

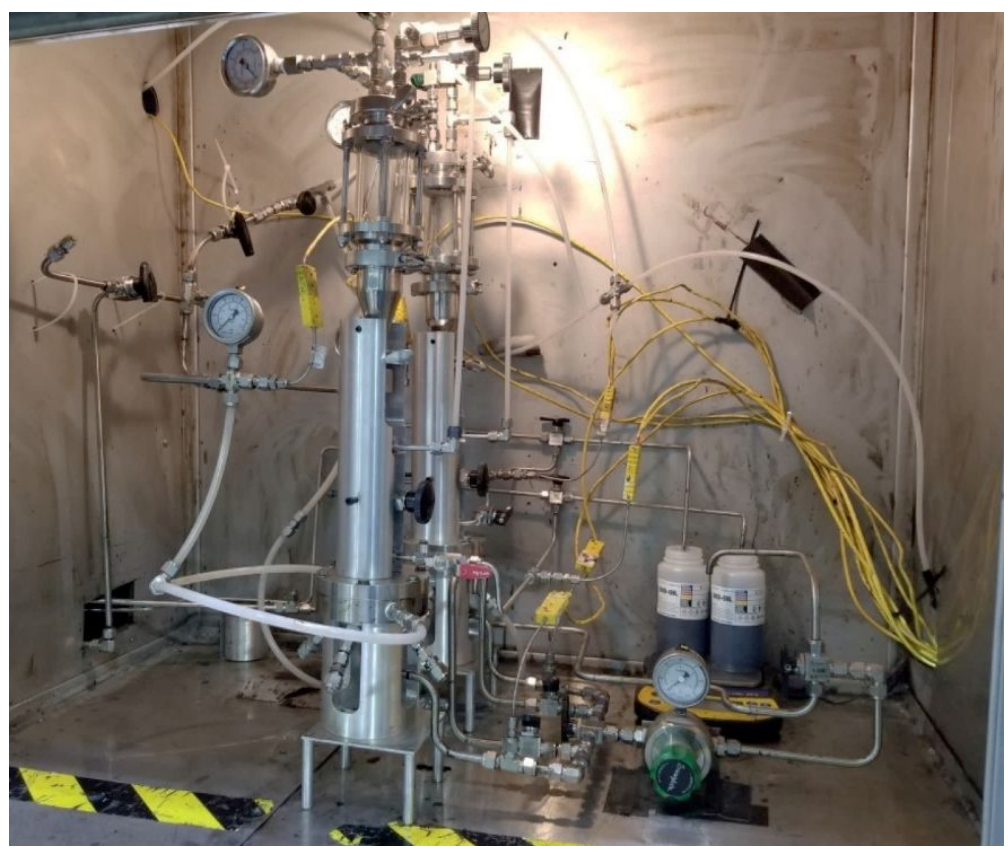

Figure S1: Picture of experimental cross-flow FPBO microfiltration system MRK III (2018)

Central to the experimental system were the customized microfiltration cells that housed the filtration media and allowed modifications to flow patterns. The filtration cells used in this work were designed 
and constructed at CanmetENERGY-Ottawa. Design features of the cells include the inversion of the feed/permeate such that the feed was introduced from the bottom of the active filtration surface (typically the permeate is at the bottom of the cell, and the retentate at the top), while the internal volume on the feed side of the cell was increased, and multiple inlet ports for fluid injection were added. The primary considerations for these design changes included the intention to facilitate the removal of the accumulated fouling on the active filtration cell and increase the shear force acting on the fouling layer. A drawing and some additional images of microfiltration cell 3 are included below.

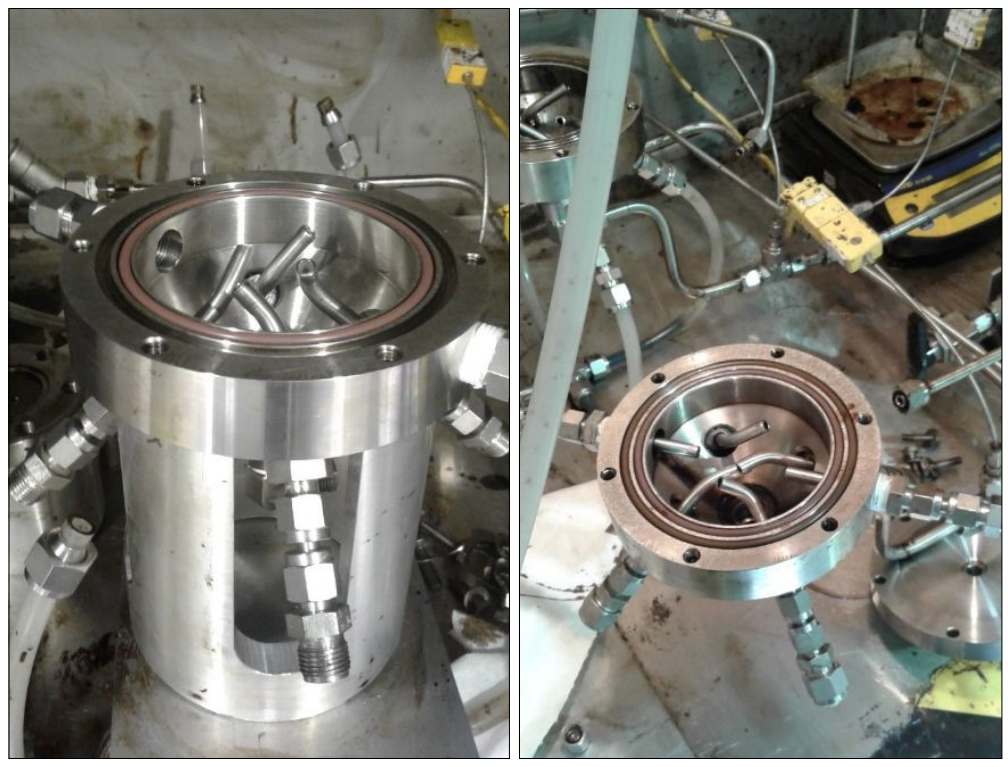

Figure S2: Side view of different flow configurations for prototype filtration cell used in bio-oil microfiltration studies with the MRK II and MRK III experimental system 
Smallest possible overall diameter to fit in $1 / 4$ " FNPT tap for fasteners

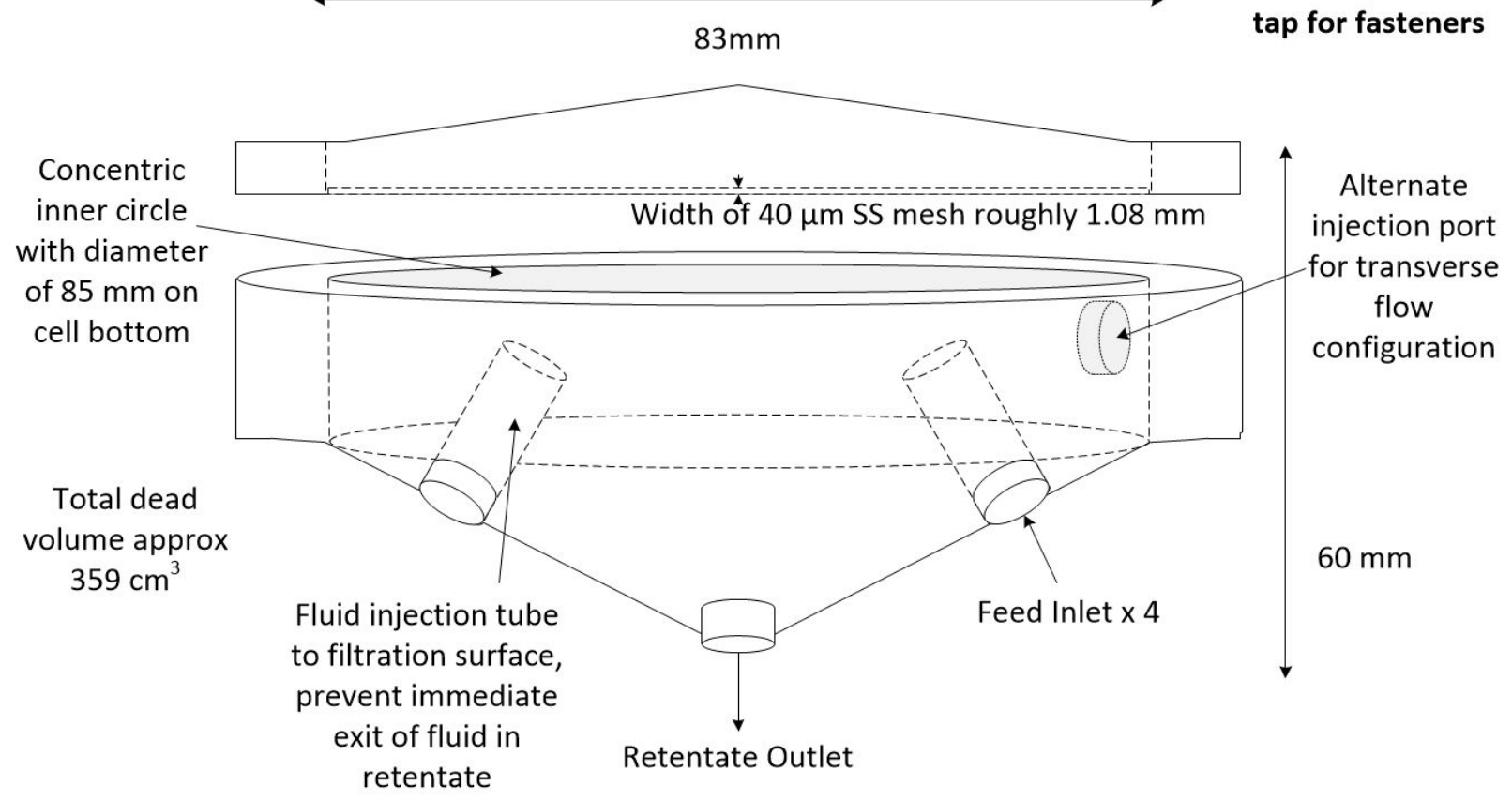

Figure S3: Simplified drawing of customized filtration cell fabricated at CanmetENERGY-Ottawa for bio-oil microfiltration studies

More details related to the design of the cross-flow microfiltration system are available elsewhere [1]. 


\section{Properties of Feedstock and Bio-oils used in Microfiltration Study}

The properties of the bio-oils used in this work are summarized in Table S1.The properties of the hardwood flooring sawdust residue are also included in Table S2.

Table S1: Summary of production information and properties of FPBOs used for microfiltration studies

\begin{tabular}{|c|c|c|c|}
\hline Internal Numerical ID & 7 & 10 & 11 \\
\hline Feedstock Origin & $\begin{array}{l}\text { Hardwood Flooring } \\
\text { Sawdust Residue }\end{array}$ & $\begin{array}{l}\text { Hardwood Flooring } \\
\text { Sawdust Residue }\end{array}$ & $\begin{array}{l}\text { Hardwood Flooring } \\
\text { Sawdust Residue }\end{array}$ \\
\hline $\begin{array}{l}\text { Approximate Reactor } \\
\text { Temperature, }{ }^{\circ} \mathrm{C}\end{array}$ & $400-480$ & $400-480$ & $460-480$ \\
\hline Age at use, months & $2-12$ & 36 & $2-36$ \\
\hline $\begin{array}{l}\text { Solids Content }{ }^{a} \text {, wt } \% \\
\text { (ASTM D7579) }\end{array}$ & 0.45 & $0.37-0.48$ & $0.35-0.63$ \\
\hline $\begin{array}{l}\text { Ash Content, wt\% } \\
\text { (ASTM D482) }\end{array}$ & 0.13 & 0.065 & 0.191 \\
\hline $\begin{array}{c}\text { Kinematic Viscosity, } \\
\mathrm{mm}^{2} / \mathrm{s} @ 40^{\circ} \mathrm{C} \\
\text { (ASTM D445) }\end{array}$ & 29.37 & 36.24 & \\
\hline $\begin{array}{l}\text { Water Content, wt\% } \\
\text { (ASTM E203) }\end{array}$ & 22.2 & 21.9 & \\
\hline $\begin{array}{l}\text { Carbon, wt\% } \\
\text { (ASTM D5291) }\end{array}$ & 46.0 & 45.1 & \\
\hline $\begin{array}{l}\text { Hydrogen, wt\% } \\
\text { (ASTM D5291) }\end{array}$ & 8.06 & 7.8 & \\
\hline $\begin{array}{l}\text { Nitrogen, wt\% } \\
\text { (ASTM D5291) }\end{array}$ & 0.38 & 0.8 & \\
\hline $\begin{array}{l}\text { Oxygen, wt } \% \\
\text { (by difference) }\end{array}$ & 45.4 & 46.3 & \\
\hline $\begin{array}{l}\text { Density, } \mathrm{kg} / \mathrm{m}^{3} \\
\text { (ASTM D4052) }\end{array}$ & 1172 & 1190 & \\
\hline $\begin{array}{c}\text { Gross Calorific Value, } \\
\text { MJ/kg } \\
\text { (ASTM D240) }\end{array}$ & 19.33 & 19.38 & \\
\hline
\end{tabular}


Table S2: Properties of hardwood flooring sawdust residue. Values include a range from 3-5 different samples

\begin{tabular}{|c|c|c|}
\hline Property & Method & Value \\
\hline Moisture Content, mf & Oven-dry (105 ${ }^{\circ} \mathrm{C},>8$ hours) & $0.03-0.08$ \\
\hline Ash Content, mf (dry basis) & ASTM D7582 & $0.003-0.015$ \\
\hline Folatile Content, mf (dry basis) & ISO 562 & $0.81-0.85$ \\
\hline Carbon, mf (dry, ash-free basis) & ASTM D7582 & $0.14-0.17$ \\
\hline Hydrogen, mf (dry, ash-free basis) & ASTM D5373 & $0.49-0.51$ \\
Nitrogen, mf (dry, ash-free basis) & ASTM D5373 & $0.06-0.07$ \\
\hline Oxygen, mf (dry, ash-free basis) & ASTM D5373 & $0.001-0.011$ \\
\hline Gross Calorific value, MJ/kg (dry) & By difference & $0.42-0.45$ \\
\hline Acetone Extractives, mf (dry) & ISO 1928 & $18.5-19.9$ \\
\hline Cellulose, mf (dry) & TAPPI T-204 & 0.017 \\
\hline Hemicellulose, mf (dry) & & 0.386 \\
\hline Lignin, mf (dry) & & 0.223 \\
\hline
\end{tabular}




\section{Properties of Filtration Media}

The properties of the various filtration media that were used over the course of the bio-oil microfiltration experiments are listed in Table S3 for reference.

Table S3: Specifications of filtration media used in experimental testing for bio-oil microfiltration

\begin{tabular}{|c|c|c|c|}
\hline Filtration Media & $\begin{array}{c}\text { Sintered Stainless } \\
\text { Steel }\end{array}$ & Twilled Stainless Steel & Nylon \\
\hline Manufacturer & BOPP & W.S. Tyler & Sterlitech \\
\hline Reference & {$[2]$} & [3] & [4] \\
\hline Nominal Pore size $(\mu \mathrm{m})$ & 1 & 40 & 5 \\
\hline Abbreviation & $1 \mu \mathrm{m} \mathrm{SS}$ & $40 \mu \mathrm{m}$ SS & $5 \mu \mathrm{m}$ nylon \\
\hline Nominal Weight $\left(\mathrm{mg} \mathrm{cm}^{-2}\right)$ & $779.3^{d}$ & 332.9 & 6.1 \\
\hline Nominal Thickness $(\mu \mathrm{m})$ & 2305 & 1219 & $65-125$ \\
\hline $\begin{array}{l}\text { Maximum Temperature } \\
\left({ }^{\circ} \mathrm{C}\right)\end{array}$ & $>200$ & $>200$ & 180 \\
\hline $\begin{array}{l}\text { Pure Water Permeance } \\
\left(\mathrm{L} \mathrm{h} \mathrm{h}^{-1} \mathrm{~m}^{-2} \mathrm{bar}^{-1}\right)\end{array}$ & $\mathrm{N} / \mathrm{A}$ & $N / A$ & $2.92 \mathrm{E}+05$ \\
\hline $\begin{array}{l}\text { Pure Air permeance } \\
\quad\left(\mathrm{L} \mathrm{h}^{-1} \mathrm{~m}^{-2} \mathrm{bar}^{-1}\right)\end{array}$ & N/A & N/A & N/A \\
\hline Bubble point (psi) & & & $6^{\mathrm{b}}$ \\
\hline Number pores per $\mathrm{m}^{2}$ & $7.00 \mathrm{E}+11$ & $2.39 E+08$ & $4.33 E+10$ \\
\hline Porosity & 0.55 & 0.3 & 0.85 \\
\hline Assumed Thickness $(\mu \mathrm{m})$ & 2305 & 1219 & 152.4 \\
\hline $\begin{array}{l}\text { Estimated Clean Filter } \\
\text { Resistance }{ }^{\mathrm{c}}\left(\mathrm{m}^{-1}\right)\end{array}$ & $1.34 \mathrm{E}+11$ & 8.13E+07 & $2.29 E+08$ \\
\hline
\end{tabular}




\section{Properties of Fouling Residues}

In the main manuscript, the use of fouling accumulation factors aided in understanding the properties of the fouling residue relative to the properties of the bulk FPBO. The data that was used to generate these values is presented below in Table S4.

Table S4: Physicochemical properties of select fouling residue samples collected from FPBO microfiltration trials

\begin{tabular}{|c|c|c|c|c|c|}
\hline & Test Method & $\begin{array}{c}\text { Fouling Residue } \\
\text { A }\end{array}$ & $\begin{array}{c}\text { Fouling Residue } \\
\text { B }\end{array}$ & $\begin{array}{c}\text { Fouling Residue } \\
\text { C }\end{array}$ & $\begin{array}{c}\text { Fouling Residue } \\
\text { D }\end{array}$ \\
\hline \multicolumn{6}{|c|}{ Bio-oil Properties (if available) } \\
\hline Solids Content, wt\% & ASTM D7579 & $0.37-0.48$ & $0.5-0.64$ & 0.41 & 0.41 \\
\hline Ash Content, wt \% & ASTM D482 & & & 0.154 & 0.154 \\
\hline Water Content, wt\% & ASTM E203 & & $13.0-16.0$ & 25.2 & 25.2 \\
\hline \multicolumn{6}{|c|}{ Processing Conditions / Fouling Residue Properties (if available) } \\
\hline Microfiltration TMP, bar & & $0.14-1.02$ & $0.34-0.68$ & $0.34-0.68$ & $0.34-0.68$ \\
\hline Filtration Media Pore Size, $\mu \mathrm{m}$ & & 5 & $5-40$ & 1 & 40 \\
\hline Carbon, wt\% & ASTM D5291 & 60.3 & 59.6 & & \\
\hline Hydrogen, wt\% & ASTM D5291 & 8.86 & 8.64 & & \\
\hline Nitrogen, wt\% & ASTM D5291 & 0.15 & 0.23 & & \\
\hline Oxygen, wt \% & by difference & 30.69 & 31.53 & & \\
\hline Ash Content, wt\% & $\begin{array}{c}\text { ASTM D3174 } \\
\text { modified }\end{array}$ & 2.44 & 1.94 & 3.25 & 2.88 \\
\hline Solids Content, wt\% & ASTM D7579 & 26.24 & 20.87 & 27.1 & 27.1 \\
\hline Water Content, wt\% & $\begin{array}{l}\text { ASTM E203 } \\
\text { modified }\end{array}$ & & & 17.4 & 18.7 \\
\hline Density, $\mathrm{kg} / \mathrm{m}^{3}$ & in-house & 1158.4 & & 1208.5 & \\
\hline \multicolumn{6}{|c|}{ Ash composition by XRF (ASTM D4326). Units = g / $100 \mathrm{~g}$ of ash } \\
\hline Silicon & ASTM D4326 & 19.15 & 18.02 & & \\
\hline Aluminum & ASTM D 4326 & 3.79 & 3.19 & & \\
\hline Iron & ASTM D4326 & 6.65 & 6.47 & & \\
\hline Titanium & ASTM D4326 & 0.17 & 0.17 & & \\
\hline Phosphorus & ASTM D4326 & 0.65 & 0.62 & & \\
\hline Calcium & ASTM D 4326 & 9.31 & 14.33 & & \\
\hline Magnesium & ASTM D 4326 & 9.54 & 9.35 & & \\
\hline Sulfur & ASTM D4326 & 1.02 & 0.76 & & \\
\hline Sodium & ASTM D 4326 & 1.59 & 0.27 & & \\
\hline Potassium & ASTM D 4326 & 2.16 & 2.49 & & \\
\hline Barium & ASTM D 4326 & 0.14 & 0.15 & & \\
\hline Strontium & ASTM D4326 & 0.05 & 0.05 & & \\
\hline Vanadium & ASTM D4326 & 0.01 & 0.01 & & \\
\hline Nickel & ASTM D4326 & 0.14 & 0.12 & & \\
\hline Manganese & ASTM D 4326 & 0.23 & 0.70 & & \\
\hline Chromium & ASTM D4326 & 0.18 & 0.16 & & \\
\hline Copper & ASTM D4326 & 1.40 & 0.18 & & \\
\hline Zinc & ASTM D4326 & 0.10 & 0.18 & & \\
\hline Loss on Fusion & ASTM D4326 & 0.46 & 0.67 & & \\
\hline Balance $^{a}$ & by difference & 43.25 & 42.13 & & \\
\hline
\end{tabular}

abalance includes normalized mass contribution of oxygen, since raw XRF data is presented with most elements in oxygenized form

In addition to the physicochemical properties above, some qualitative analysis of the fouling residues was performed. Firstly, the relative kinematic viscosity of the fouling residue was compared at $40^{\circ} \mathrm{C}$ and 
$60^{\circ} \mathrm{C}$, as shown in Figure S4. It was observed that there was no distinguishable qualitative variation in rheological properties at the two temperature levels.
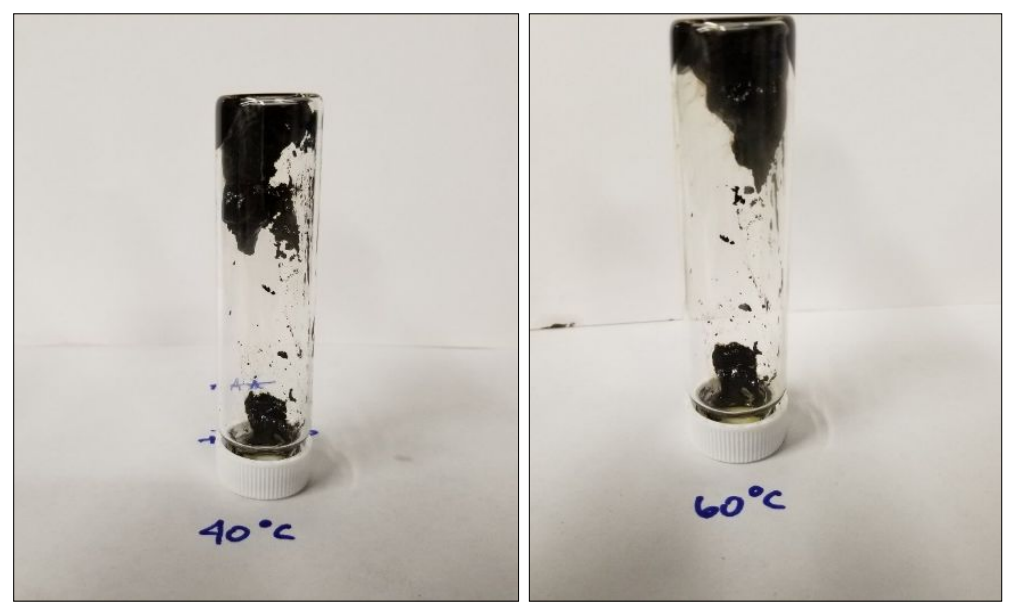

Figure S4: Fouling residue at $40^{\circ} \mathrm{C}$ (left) and $60^{\circ} \mathrm{C}$ (right)

Furthermore, the solubility of the fouling residue was qualitatively assessed using different solvents at a ratio of 5:1 (solvent: fouling residue, mass basis). The solvent and fouling residue was mixed by shaking the vial for approximately 30 seconds. Overall, it was found that qualitatively that the fouling residue demonstrated the highest relative solubility in acetone, methanol and ethanol. At the same time, it was least soluble in the diethylene glycol monomethyl ether. 


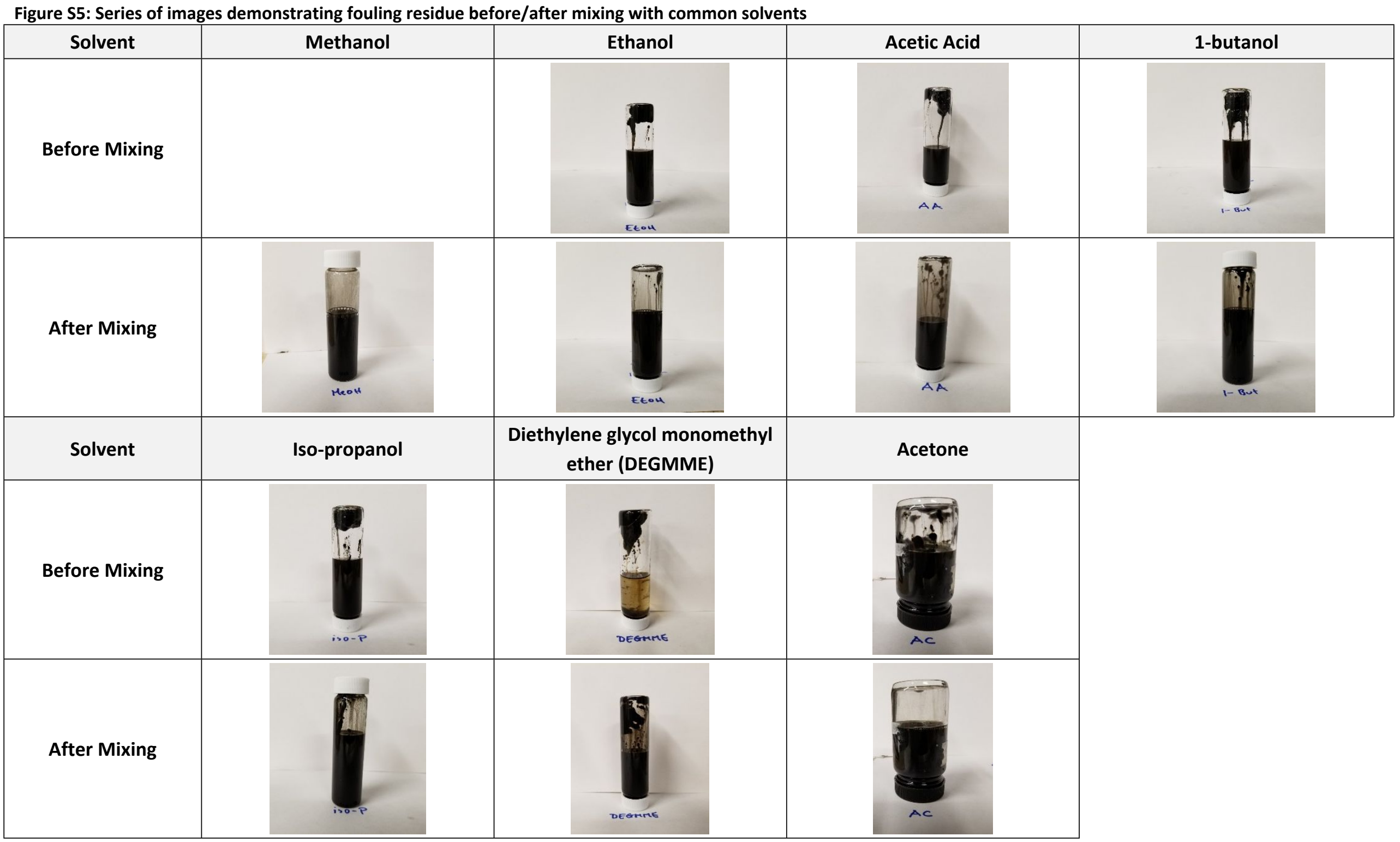


Additional Experimental Data from Intermittent Backflushing Trials

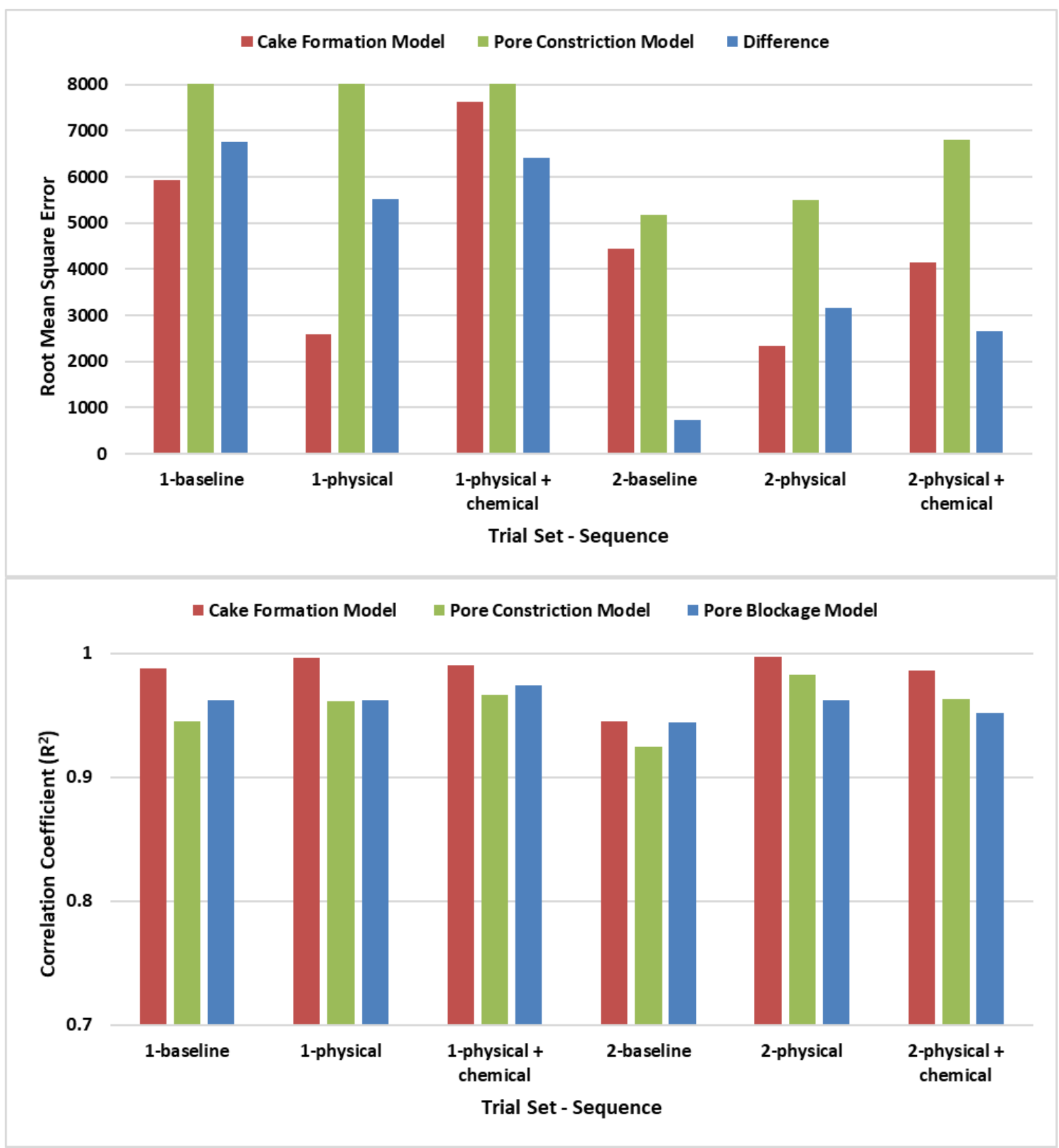

Figure S6: Root mean square error (top) and correlation coefficient (bottom) from cake formation, pore constriction models and pore blockage linear models relative to observed experimental data investigating manual regeneration of filtration media 


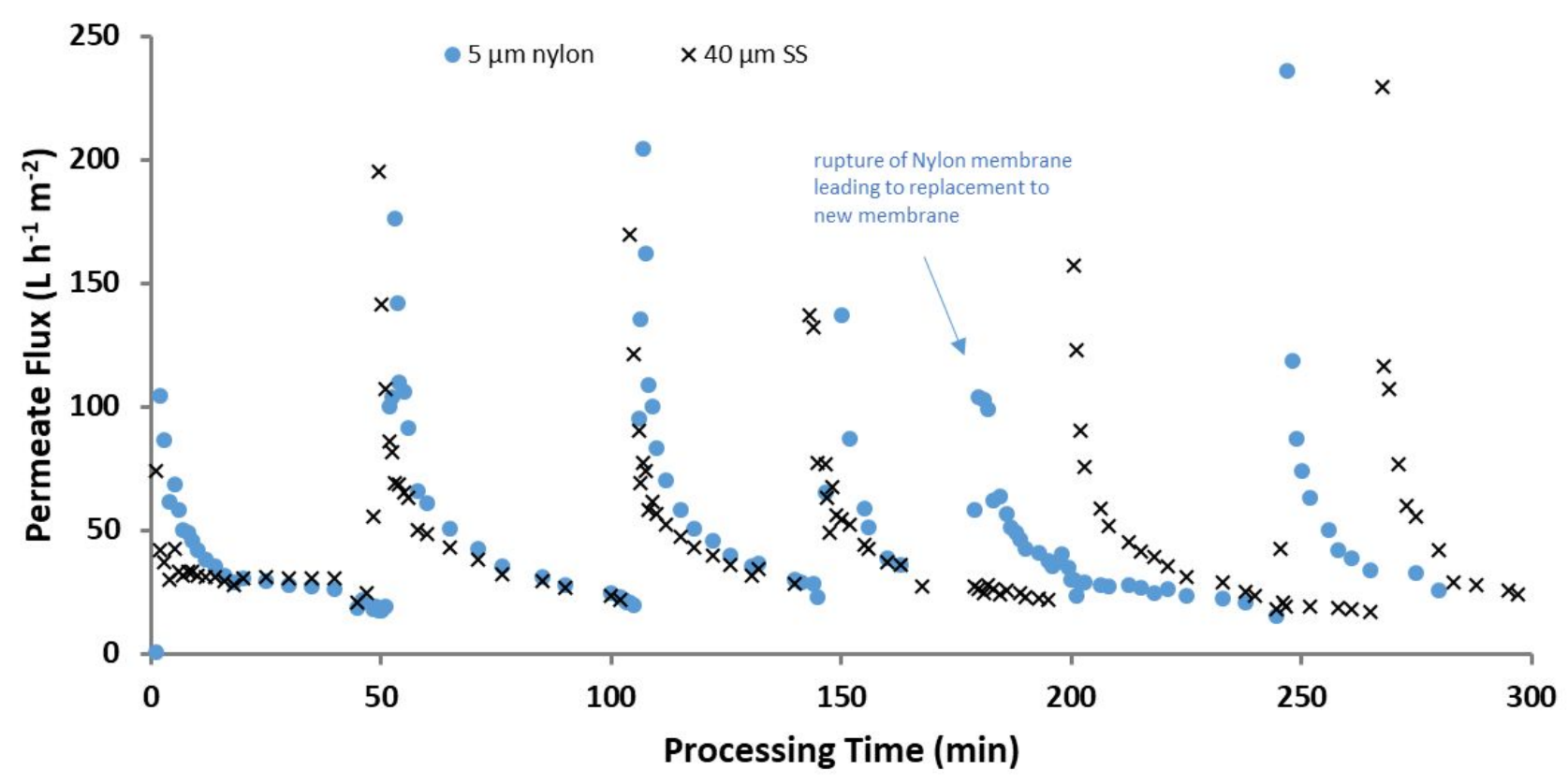

Figure S7: Permeate flux profiles as a function of FPBO cross-flow microfiltration processing time with periodic permeate backflushing

Cleaning sequence: $39.3 \mathrm{~L} \mathrm{~m}^{-2}$ air only (1.4 bar) at 50 minutes; $3.9 \mathrm{~L} \mathrm{~m}^{-2}$ permeate (1.4 bar) at room temperature at 105 minutes; $8 \mathrm{~L} \mathrm{~m}^{-2}$ permeate (1.4 bar) at room temperature at 145 minutes; $31.4 \mathrm{~L} \mathrm{~m}^{-2}$ permeate (1.4 bar) at room temperature at 200 minutes for $40 \mu \mathrm{m} \mathrm{SS} ; 8 \mathrm{~L} \mathrm{~m}^{-2}$ permeate (1.4 bar) at 60 ${ }^{\circ} \mathrm{C}$ at 250 minutes for $5 \mu \mathrm{m}$ nylon; $8 \mathrm{~L} \mathrm{~m}^{-2}$ permeate (1.4 bar) at $60{ }^{\circ} \mathrm{C}$ at 265 minutes for $40 \mu \mathrm{m} \mathrm{SS}$ 


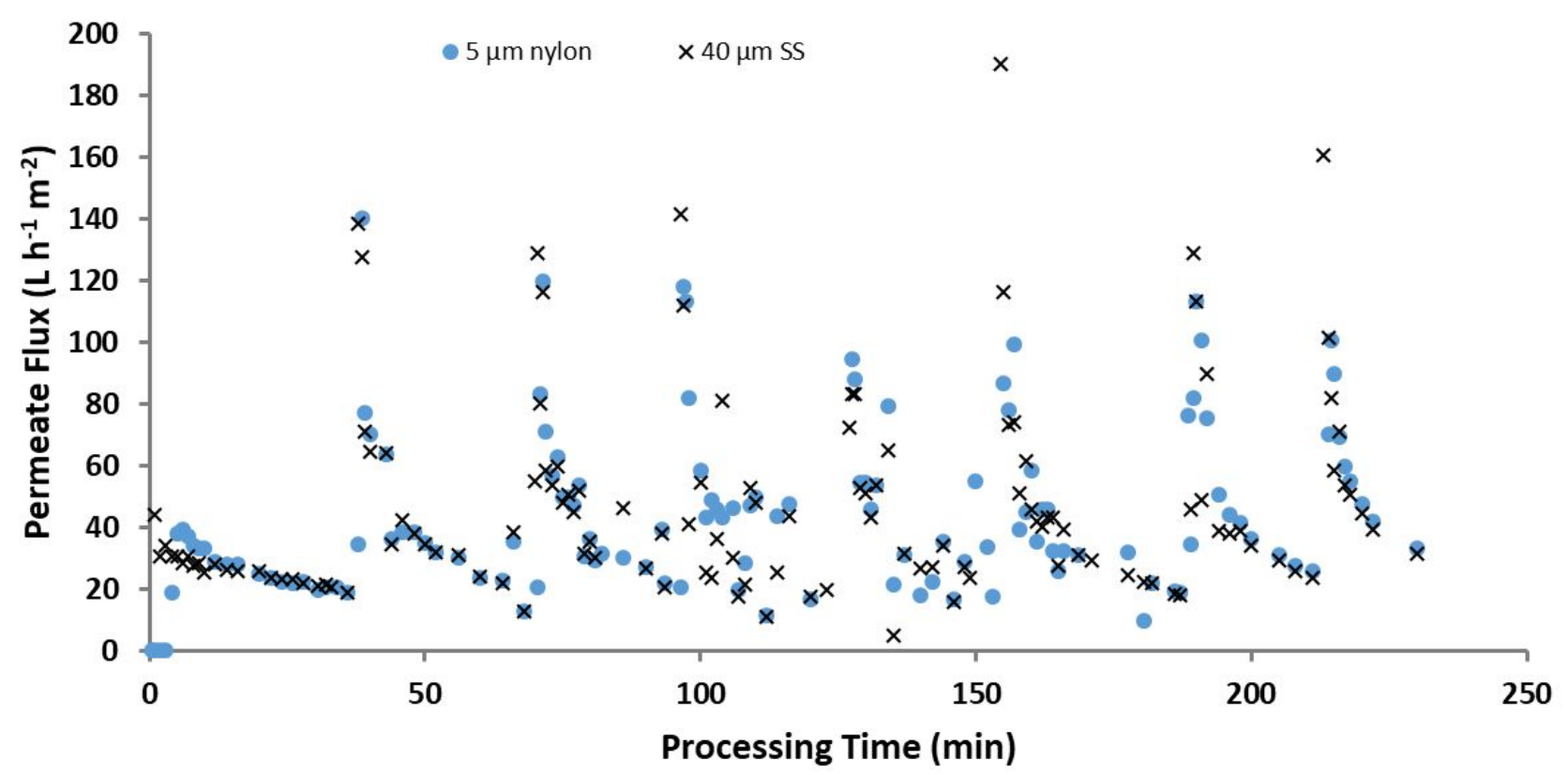

Figure S8: Permeate flux as a function of FPBO cross-flow microfiltration processing time with periodic solvent backflushing

Cleaning sequence: $40 \mathrm{~L} \mathrm{~m}^{-2}$ air only (1.4 bar) at 35 minutes; $8 \mathrm{~L} \mathrm{~m}^{-2}$ ethanol (1.4 bar) at room temperature at 70 and 100 minutes; $8 \mathrm{~L} \mathrm{~m}^{-2}$ methanol (1.4 bar) at room temperature at 125 and 150 minutes; $8 \mathrm{~L} \mathrm{~m}^{-2}$ DEGMME (1.4 bar) at room temperature at 190 and 215 minutes 


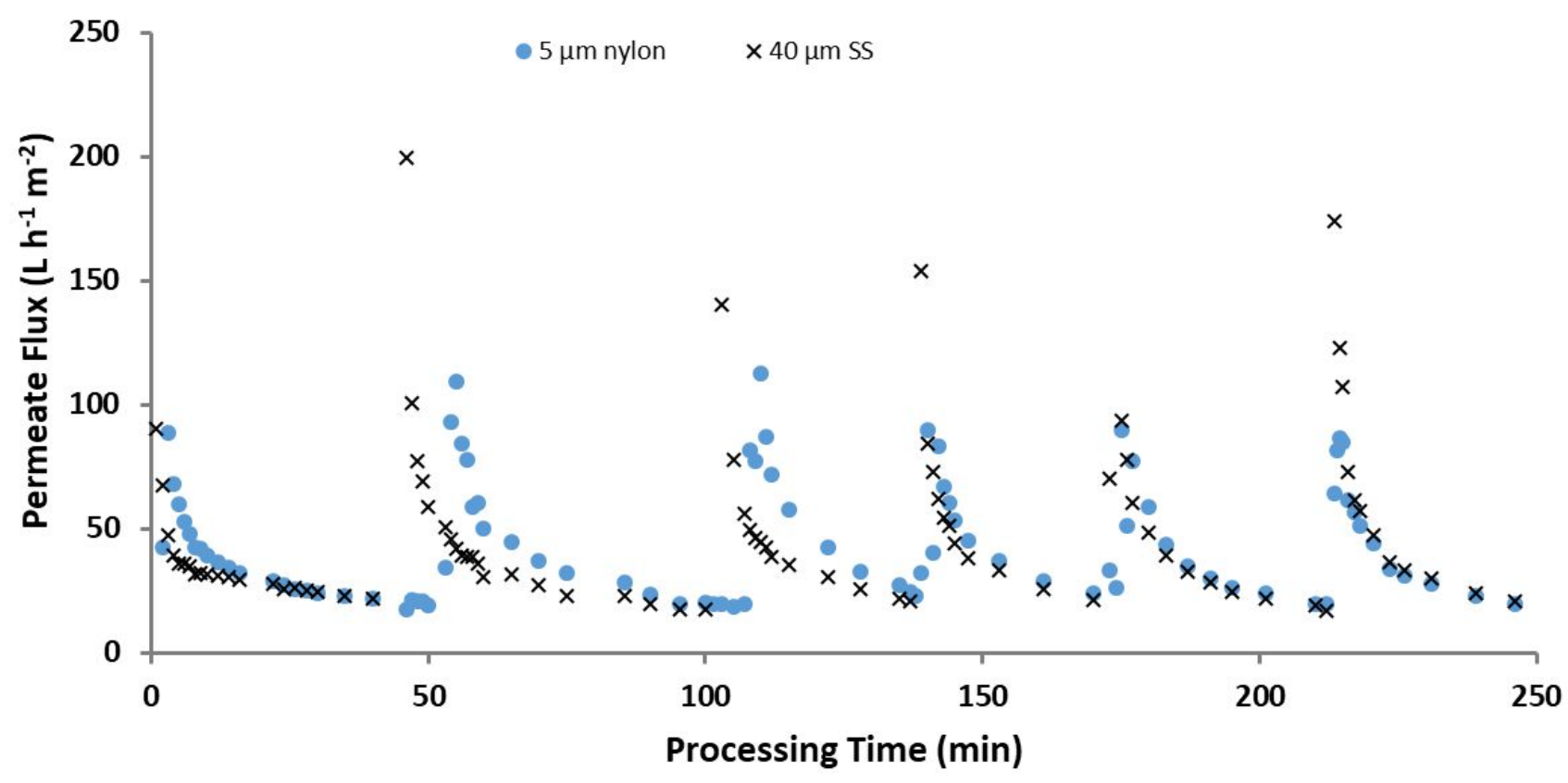

Figure 59: Permeate flux as a function of FPBO cross-flow microfiltration processing time with periodic air backflushing

Cleaning sequence: $40 \mathrm{~L} \mathrm{~m}^{-2}$ air (20 psig) at 50, 105, 140, 174 and 212 minutes 


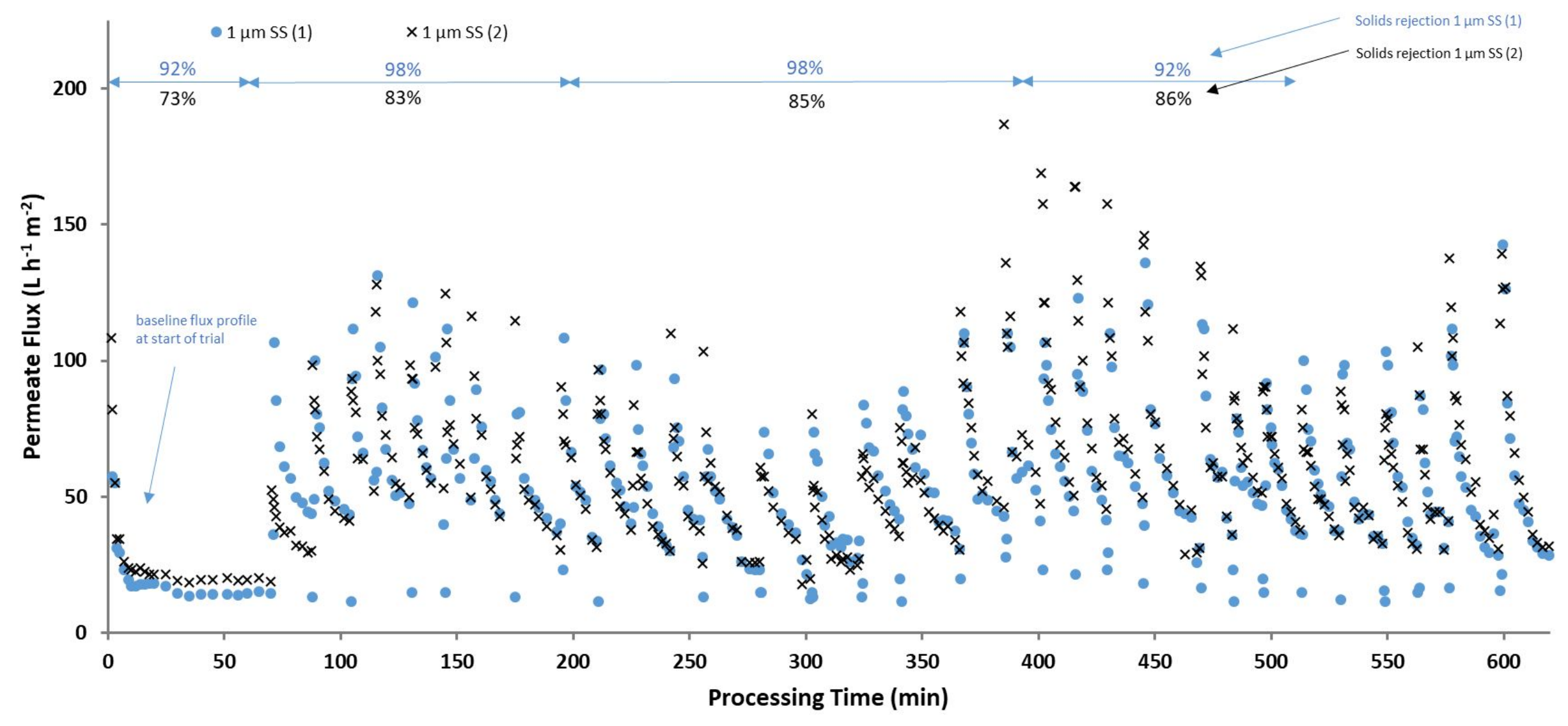

Figure S10: Permeate flux as a function of processing time with extended air backflushing

Cleaning sequence: $39.3 \mathrm{~L} \mathrm{~m}^{-2}$ air (20 psig) at ambient temperature at 70, 88, 104, 115, 130, 156, 175, 195, 210, 225, 242, 256, 280, 302, 323, 340, 366, 385, 401, $416,430,445,470,483,496,513,530,548,563,577,598$ and 623 minutes. 

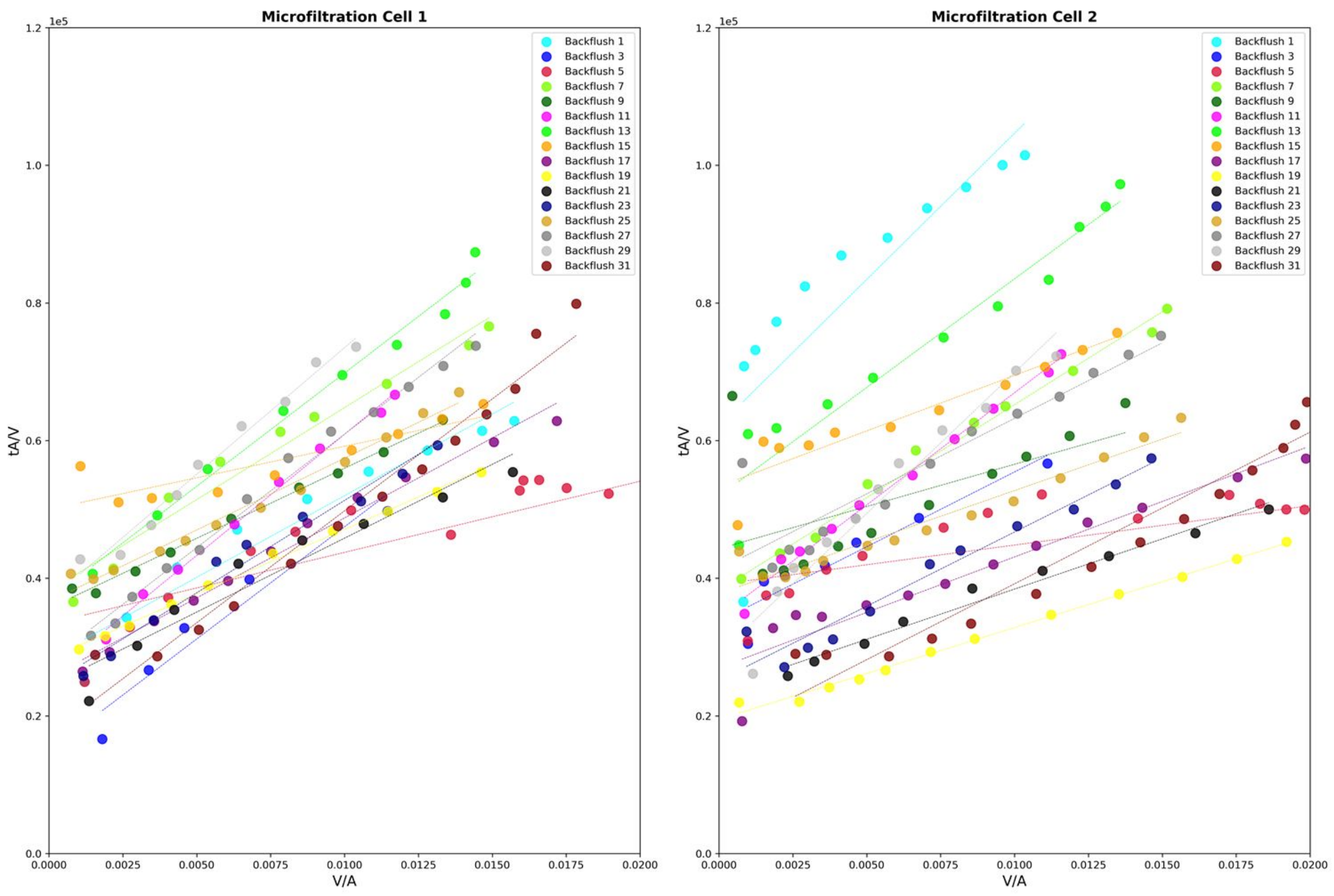

Figure S11: Linearized resistance model for cake formation, data extracted from Figure S10 

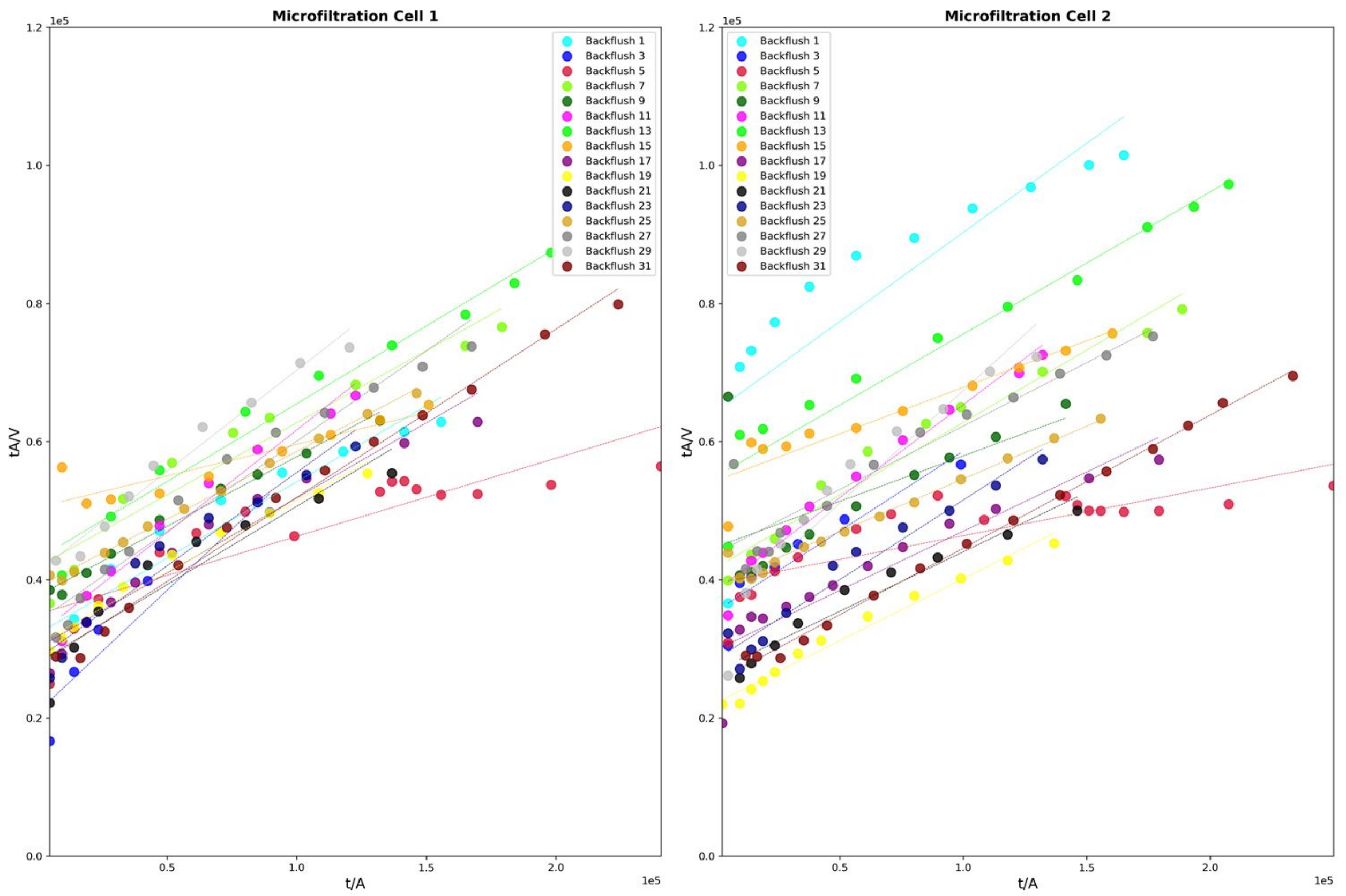

Figure S12: Linearized resistance model for pore constriction, data extracted from Figure S10 

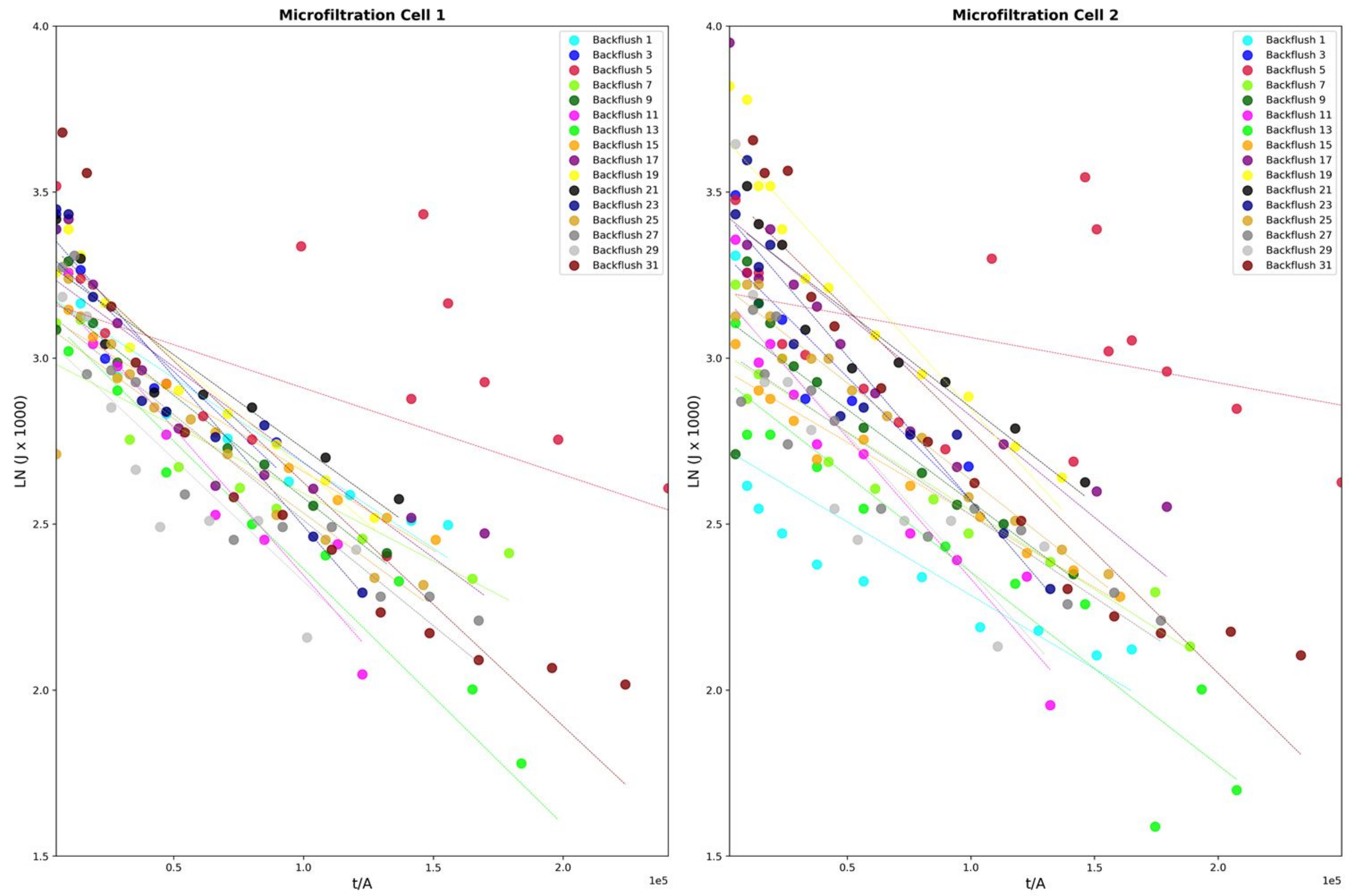

Figure S13: Linearized resistance model for pore blockage, data extracted from Figure S10 


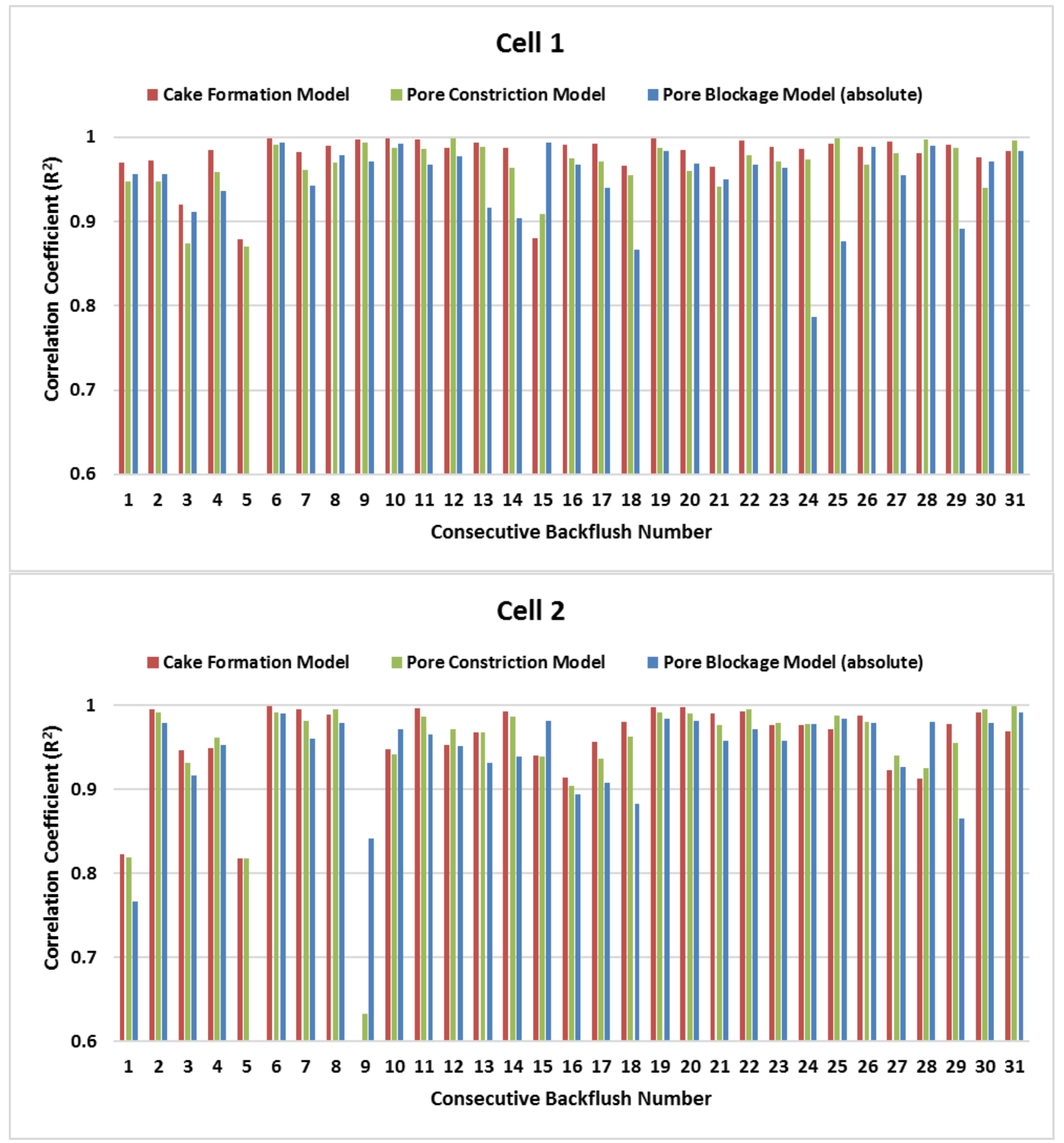

Figure S14: Calculated correlation coefficient $\left(R^{2}\right)$ for cake formation, pore constriction and pore blockage models as function of consecutive backflush number for cell 1 (top) and cell 2 (bottom) from extended air backflushing trial 


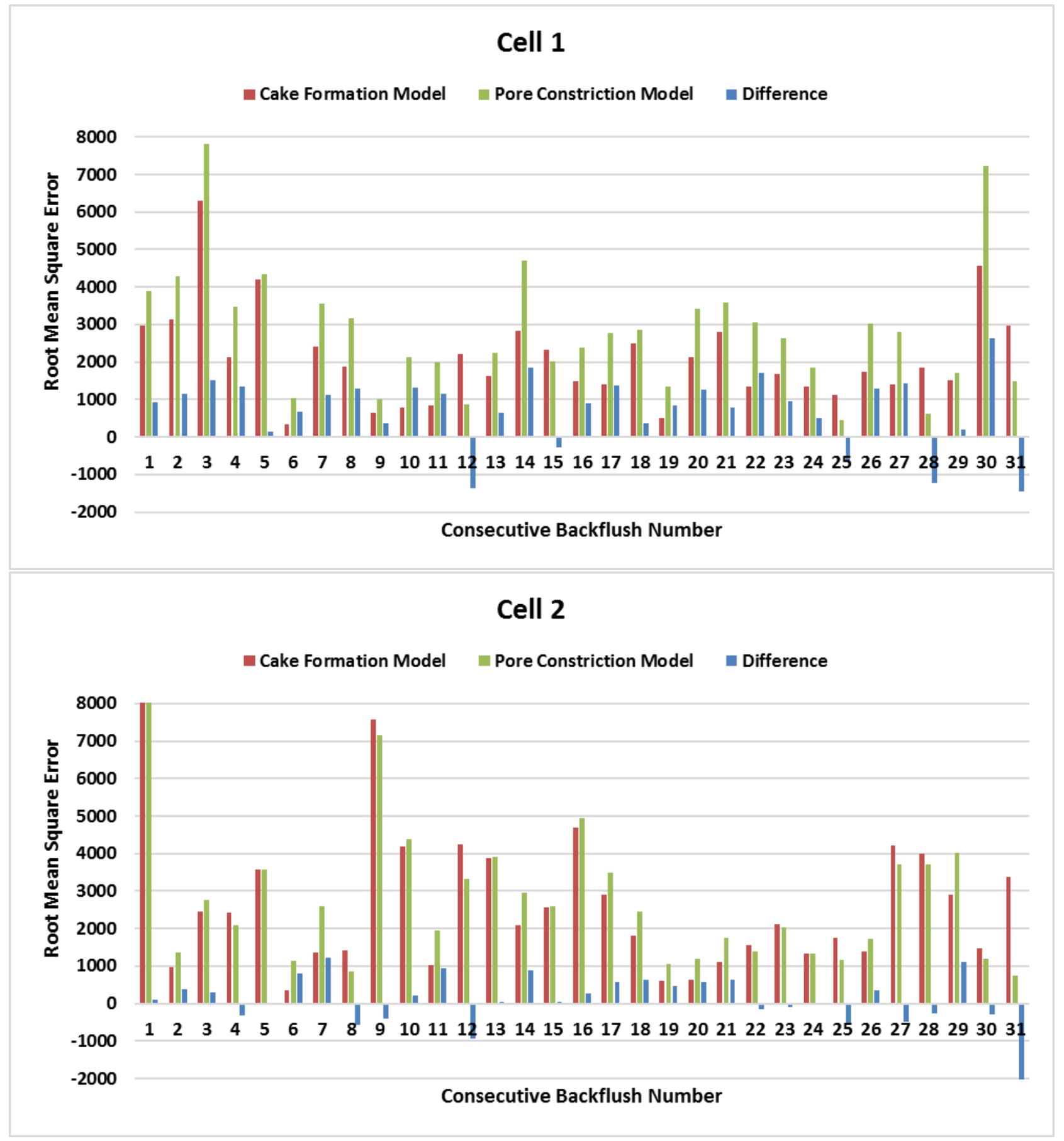

Figure S15: Root mean square error of cake formation and pore constriction models, and difference between the two, as function of consecutive backflush number for cell 1 (top) and cell 2 (bottom) from extended air backflushing trial 


\section{Additional References}

[1] Mazerolle, D. Experimental Evaluation of Solids and Ash Removal Pathways of Fast Pyrolysis BioOils. Master's Dissertation, University of Ottawa, Ottawa, ON, 2019. https://doi.org/https://dx.doi.org/10.20381/ruor-24122.

[2] BOPP, Precision woven stainless steel filter mesh. https://www.bopp.com/logpdf.aspx?filepath=DownloadCenter/English/brochures/Bopp_FI_Metallfiltergewebee_2015_WEB.pdf\&filetitel=BOPP\%20FI\%20Filtermeshes (accessed March 2020)

[3] W.S Tyler, Wire cloth handbook. https://wstyler.com/downloads/wire-cloth-handbook/ (accessed March 2020)

[4] Sterlitech, Nylon Membrane Filters. http://www.sterlitech.com/filters/membrane-discfilters/nylon-membranes.html (accessed December 2019)

[5] Davis R.H., Grant D.C. Theory for Deadend Microfiltration. In Membrane Handbook; Ho W.S.W., Sirkar K.K., Eds.; Springer, Boston, MA, 1992; pp 461-479. https://doi.org/10.1007/978-1-46153548-5_32 